\title{
A new type of Hypersensitivity Pneumonitis: salami brusher's disease
}

\author{
M. Marvisi, L. Balzarini, C. Mancini, P. Mouzakiti
}

\begin{abstract}
A new type of Hypersensitivity Pneumonitis: salami brusher's disease. M. Marvisi, L. Balzarini, C. Mancini, P. Mouzakiti.

We observed five consecutive cases of Hypersensitivity Pneumonitis in subjects working in a salami factory. The workers had to clean the white mould growing on salami surface using a manual wire brush. The five patients (four female) had a mean age of $39 \pm 15$ years; two were smokers. Three patients had an acute clinical presentation with fever, dyspnoea, dry cough, oxygen desaturation, and presented at the emergency department with suspected diagnosis of community acquired pneumonia. The mean latency for developing respiratory symptoms was 11.6 days. Pulmonary function test demonstrated a reduction in diffusing capacity (DLCO) in all 5 patients $(60 \pm 15 \%$ of predicted value). Skin prick test was positive
\end{abstract}

\begin{abstract}
for Penicillium spp in 3 cases and for Cladosporium and Aspergillus spp in 2 others. Specific IgG antibodies against Penicillium spp were positive in 3 subjects; 2 were positive for Aspergillus Fumigatus. The prevailing radiological pattern was a ground glass appearance in the three patients with acute clinical onset and a centrilobular one in patients with subacute onset. All patients were advised to avoid exposure to the antigens. Follow-up visits including pulmonary function testing, and DLCO measurement were conducted at one, three and six months. HRCT was performed at six month. Four subjects had a complete radiological and clinical resolution after changing work. Only one patient was treated with oral steroids for severe dyspnoea and progressive reduction of DLCO, gaining a complete radiological and clinical stability at six months. Monaldi Arch Chest Dis 2012; 77: 1, 35-37.
\end{abstract}

Keywords: Hypersensitivity pneumonitis, Salami Brusher's, Penicillium, Aspergillus.

Department of Internal Medicine and Pneuumology, Clinica Figlie di S. Camillo, Cremona, Italy.

Correspondence: Dr. Maurizio Marvisi, Director Department of Internal Medicine and Pneuumology, Clinica Figlie di S.Camillo,Via Filzi 56, 26100 Cremona, Italy; e-mail:mmarvis@alice.it

Hypersensitivity Pneumonitis (HP) is a protean syndrome of varying intensity, clinical presentation and natural history. It represents an immunologic reaction to an inhaled organic antigen. Initially it was associated with farming. With time, a large variety of environmental settings and antigens have been described including, but not limited to, agricultural dusts, bioaerosols, avian antigens, drugs and certain reactive chemical species that form haptens.

Its prevalence and incidence are thought to be low, although few population-based or real cohort studies have been conducted to address this question, and many patients with mild or subclinical HP escape detection or are misdiagnosed as suffering from viral illness or asthma [1].

\section{Case reports}

We observed 5 consecutive cases of HP in subjects working in a salami factory located in the Emilia-Romagna region in the province of Piacenza (Northern Italy). The factory won a contract to supply products for the German market, and the workers had to clean thoroughly the white mould growing naturally on the salami surface, using a manual wire brush. They did this task daily for eight hours a day without respiratory protection, and were constantly exposed to high levels of brushing dust.
The study includes five patients (4 females), with a mean age of $39 \pm 15$ years; two of them were smokers $(40 \%)$. The total working population consisted of 30 subjects (29 female), mean age $40 \pm 15$, 5 of whom were smokers. The prevalence of HP was $17 \%$.

Three patients had an acute clinical presentation (fever, dyspneoa, dry cough, oxygen desaturation), and presented at the emergency department with suspected diagnosis of community acquired pneumonia. The mean latency (exposure time in days until onset of symptoms) was 11.6 days. At the time of diagnosis, a complete clinical history was taken, and radiologic studies, pulmonary function test, blood analysis, fiberoptic bronchoscopy with bronchoalveolar lavage and immunologic studies were performed (table 1). Pulmonary function test demonstrated a reduction in diffusing capacity of the lung (DLCO) in all 5 patients ( $60 \pm 15 \%$ of predicted value). A bronchial hyperresponsiveness to methacoline was present in all the patients, the mean dose of methacoline causing a $20 \%$ fall in $\mathrm{FEV}_{1}$ being $3.63 \pm 4.70 \mathrm{mg} / \mathrm{mL}$. For all patients, specific IgG antibodies were determined by an enzyme-linked immunoabsorbent assay (ELISA) technique (Siemens AG. Germany).

Antibodies against Penicillium spp were positive in 3 patients; 2 patients were positive to $A s$ pergillus Fumigatus. Skin prick test (Lofarma. Mi- 
Table 1. - Demographic, clinical and instrumental characteristics of patients

\begin{tabular}{|c|c|c|c|c|c|}
\hline Patients & 1 & 2 & 3 & 4 & 5 \\
\hline Name & A.C. & B.C. & B.R. & C.C. & P.C. \\
\hline Sex & $\mathrm{F}$ & $\mathrm{F}$ & M & F & $\mathrm{F}$ \\
\hline Age & 46 & 54 & 49 & 39 & 36 \\
\hline History & Neg. & Allergic rhinitis & Neg. & Neg. & Asthma, rhinitis \\
\hline Symtoms & Fever, cough & Malaise, fever & Fever, dry cough & Fever, dyspnea & Asthenia, dyspnea, fever \\
\hline Skin test & Penicillum & Clados., asperg. & Penicillum & Penicillum & Cladosporium, aspergillus \\
\hline Serum Precipitins & Penicillum & Asperg. & Penicillum & Penicillum & Asperg. \\
\hline B.A.L. & L.: 84\%; CD4/CD8 0.43 & L: $57.5 \%$; CD8: $60 \%$ & L: 40\%; CD4/CD8 0.56 & L: $84 \%$ & L: $74 \%$ \\
\hline Dlco & $45 \%$ del pred & $60 \%$ & $62 \%$ & $60 \%$ & $70 \%$ \\
\hline $\mathrm{FEV}_{1}$ & $95 \%$ del pred & $90 \%$ & $75 \%$ & $85 \%$ & $75 \%$ \\
\hline $\mathrm{MEF}_{25-75}$ & Norm. & $50 \%$ del pred & $35 \%$ & Norm. & $65 \%$ \\
\hline Smoking habit & No & 36 Packs/years & 40 Packs/years & No & No \\
\hline Latency & 8 Days & 2 Weeks & 3 Weeks & 5 Days & 10 Days \\
\hline $\begin{array}{l}\text { Radiography and } \\
\text { HRCT }\end{array}$ & $\begin{array}{l}\text { Lower lobes } \\
\text { Infiltrates }\end{array}$ & $\begin{array}{l}\text { Ground glass } \\
\text { Centrilobular pattern }\end{array}$ & $\begin{array}{l}\text { Centrilobular pattern } \\
\text { Ground glass Nodular pattern }\end{array}$ & Ground Glass & Centrilobular pattern \\
\hline
\end{tabular}

lano. Italy) was positive for Penicillium spp in 3 cases and for Cladosporium spp and Aspergillus spp in 2 others; patients 2 and 5 were positive to both Aspergillus and Cladosporium (table 1) at High Resolution Computed Tomography (HRCT).

The prevailing radiological pattern was a ground glass appearance in the three patients with acute onset and a centrilibular one in patients with subacute onset (table 1, figure 1).

All patients were advised to avoid exposure to the antigens. Follow-up visits including pul-

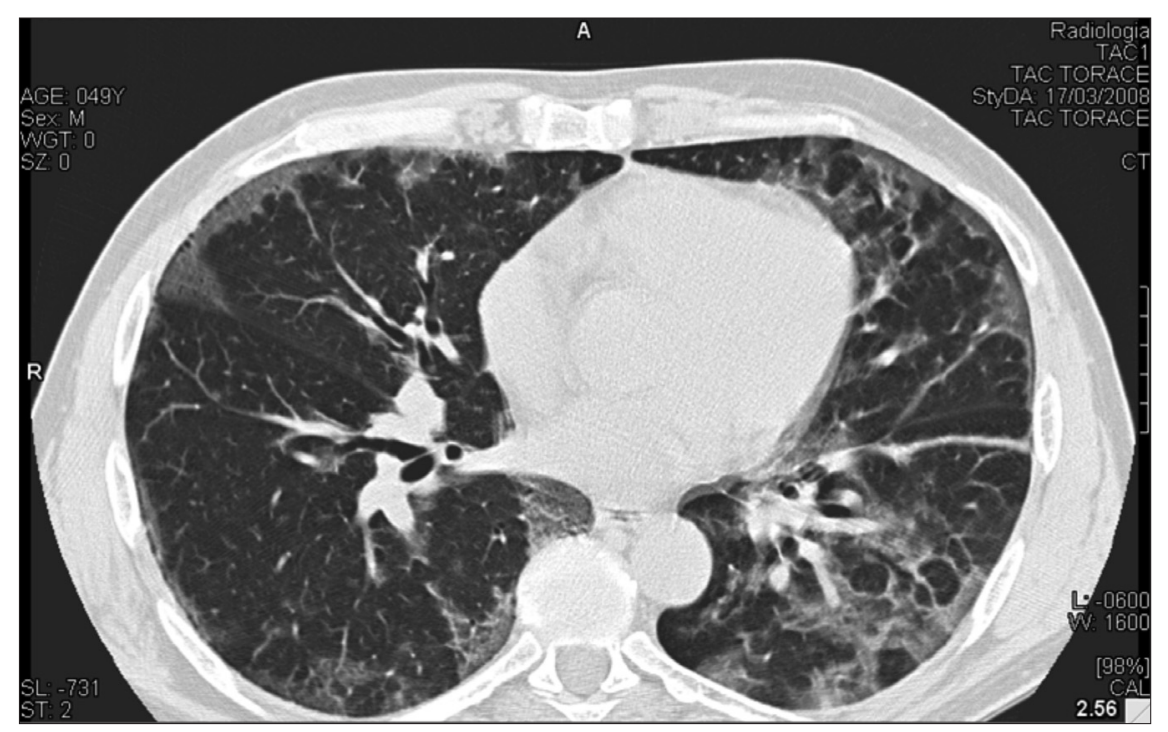

Fig. 1. - HRCT of one patient showing bilateral infiltrates. monary function testing, and DLCO measurements were conducted at one, three and six months. HRCT was performed at six months. Four patients had a complete radiological and clinical resolution after changing work. Patient 3 was treated with oral steroids $(0.5 \mathrm{mg} / \mathrm{Kg})$ for severe dyspnoea and progressive reduction in DLco, gaining radiological and clinical stability at six months.

The rest of the working population consisted of 25 females whose mean age was $40 \pm 15$ years, five of whom were smokers (prevalence $25 \%$ ). They were free of pulmonary symptoms and had a negative history for COPD, asthma or other pulmonary disorders. Pulmonary function tests including methacoline test and standard chest $\mathrm{X}$-ray were normal in all subjects. Skin prick test was negative. The determination of specific IgG antibodies was positive in 4: 2 were positive to $A s$ pergillus spp, 1 for Penicillum spp and 1 for Cladosporium.

\section{Discussion}

Salamis, sausages, pork neck salamis and culatello are full of prokaryotic organisms including bacteria (Pseudomonas, Acinetobac- 
ter, etc.), yeasts (Saccharomyces, Trichosporon, etc.) and moulds (Penicillium, Aspergillus, Cladosporium, Candida) [2, 3].

In the English language literature there are few case reports of HP due to sausage casing dust. In a recent report, Morell et al. described five Spanish patients working in a food factory involved in the production of dry sausage. In that series, specific immunoglobulins to Penicillium frequentans and Aspergillus fumigatus were positive in three patients. Only one of them was a current smoker, and three patients had an acute clinical onset. A few years ago, Guillot et al. described three non-smoking patients: one of them had an acute clinical presentation, and two of them were treated with steroids with complete resolution of symptoms. Precipitins to Penicillium Nalgiovense were positive in all cases $[2,3]$.

Our series confirms the key role of Penicillium and Aspergillus in the genesis of the disease, and provides additional information. The prevalence in the working population seems to be high (17\%). The majority of patients had an acute clinical presentation with fever, cough and oxygen desaturation, based on the follow-up findings. This type of HP appears to have a good prognosis if patients avoid exposure to causative agents.

In addition, our small series of cases would seem to contradict previous epidemiological data showing that HP occurs rarely in non-smokers and usually presents with an insidious clinical onset.
The mechanism for this effect is probably due to the fact that cigarette smoke depresses both $\mathrm{T}$ cell independent and $\mathrm{T}$ cell dependent responses to inhaled antigens. In this study 2 of the five patients were current smokers (prevalence of 40\%) and 1 of them had an acute clinical onset. The probable mechanism is due to the fact that the cell wall of moulds such as Penicillium spp and others contains a large amount of a particular polyglucose compound: the $\beta-1,3$ glucan. This compound can attach to human cells through the dectin-1 receptor, which is present in myeloid cells such as macrophages, dendritic cells, and neutrophils, inducing activation of $\mathrm{Nf} \kappa \mathrm{B}$, which initiates the production of several inflammatory cytokines [4]. Further immunological and epidemiological data is therefore warranted to confirm this hypothesis.

\section{References}

1. Girard M, Cormier Y. Hypersensitivity Pneumonitis. Curr Opin Allergy Clin Immunol 2010; 10: 99-103.

2. Morell F, Cruz MJ, Gomez FP, Rodriguez Jerez F, Xaubet A, Munoz X. Chacinero's lung-hypersensitivity pneumonitis due to dry sausage dust. Scand J Work Environ Health 2011; 37: 349-56.

3. Guillot M, Bertoletti L, Deygas N, Maberin H, Fareo Vergnon JM. Pneumopathie a la fleur de saucisson: trois observation. Rev Mal Respir 2008; 25: 596-600.

4. Rylander R. Organic dust induced pulmonary disease. The role of mould derived B-glucan. Ann Agric Environ Med 2010; 17: 9-13.

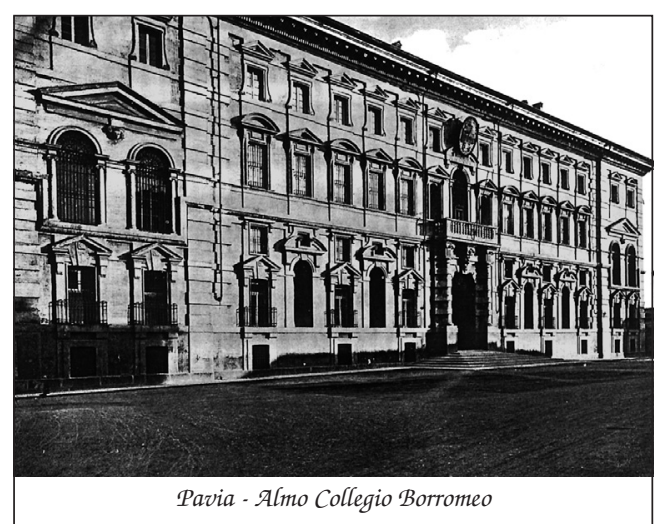

